\title{
Formalizing and Proving Privacy Properties of Voting Protocols Using Alpha-Beta Privacy
}

\section{Gondron, Sébastien Pierre Christophe; Mödersheim, Sebastian Alexander}

\section{Published in:}

Proceedings of 24th European Symposium on Research in Computer Security

Link to article, DOI:

10.1007/978-3-030-29959-0_26

Publication date:

2019

Document Version

Peer reviewed version

Link back to DTU Orbit

\section{Citation (APA):}

Gondron, S. P. C., \& Mödersheim, S. A. (2019). Formalizing and Proving Privacy Properties of Voting Protocols Using Alpha-Beta Privacy. In Proceedings of 24th European Symposium on Research in Computer Security (pp. 535-555). Springer. Lecture Notes in Computer Science Vol. 11735 https://doi.org/10.1007/978-3-030-299590_26

\section{General rights}

Copyright and moral rights for the publications made accessible in the public portal are retained by the authors and/or other copyright owners and it is a condition of accessing publications that users recognise and abide by the legal requirements associated with these rights.

- Users may download and print one copy of any publication from the public portal for the purpose of private study or research.

- You may not further distribute the material or use it for any profit-making activity or commercial gain

- You may freely distribute the URL identifying the publication in the public portal 


\title{
Formalizing and Proving Privacy Properties of Voting Protocols using Alpha-Beta Privacy
}

\author{
Sébastien Gondron and Sebastian Mödersheim \\ $\{$ spcg,samo\}@dtu.dk
}

\author{
DTU Compute, Richard Petersens Plads, Building 324, \\ 2800 Lyngby, Denmark
}

\begin{abstract}
Most common formulations of privacy-type properties for security protocols are specified as bisimilarity of processes in applied- $\pi$ calculus. For instance, voting privacy is specified as the bisimilarity between two processes that differ only by a swap of two votes. Similar methods are applied to formalize receipt-freeness. We believe that there exists a gap between these technical encodings and an intuitive understanding of these properties.

We use $(\alpha, \beta)$-privacy to formalize privacy goals in a different way, namely as a reachability problem. Every state consists of a pair of formulae: $\alpha$ expresses the publicly released information (like the result of the vote) and $\beta$ expresses the additional information available to the intruder (like observed messages). Privacy holds in a state if every model of $\alpha$ can be extended to a model of $\beta$, i.e., the intruder cannot make any deductions beyond what was deliberately released; and privacy of a protocol holds if privacy holds in every reachable state.

This allows us to give formulations of voting privacy and receiptfreeness that are more declarative than the common bisimilarity based formulations, since we reason about models that are consistent with all observations like interaction with coerced (but potentially lying) voters. Also, we show a relation between the goals: receipt-freeness implies voting privacy.

Finally, the logical approach also allows for declarative manual proofs (as opposed to long machine-generated proofs) like reasoning about a permutation of votes and the intruder's knowledge about that permutation.
\end{abstract}

Keywords: formal security models, logic and verification, privacy preserving systems, voting protocols, receipt-freeness, coercion-resistance, security requirements, security protocols. 


\section{Introduction}

Privacy is essential for freedom: to make a choice like a vote in a completely free way, i.e., determined only by one's own convictions, context, interests and expectations (rather than those of others), it is crucial that this choice cannot be observed by others. However, it is not sufficient to give people the possibility to make the choice in a private way: we also have to actually prevent them from proving what they have chosen. While one has the right to say what one has chosen (by the freedom of speech), we must also guarantee the possibility to lie about it, too. The reason is that otherwise we limit the privacy through a backdoor, as there can arise the pressure to prove what one has chosen, especially when bribery or abuse of power comes into play. This paper investigates the tension between privacy and coercion with the focus on voting privacy, however, this is also relevant in other areas like electronic medical prescriptions (preventing pressure from the pharmaceutical industry onto doctors).

Related to the understanding of privacy goals is the problem that a demagogue can easily raise doubts in the legitimacy of an election. Our best chance to defeat this are open source system that scientists, and ideally also ordinary people, can understand and convince themselves to be correct. The less obscure, the harder it is to defame, and the easier to recognize criticisms as unfounded. One of the challenges for describing systems in both a formally precise and intuitive way are privacy goals and their subtle relation to coercion.

Most common formulations of privacy-type properties for security protocols are specified as bisimilarity of processes in applied- $\pi$ calculus [1 [4], and we consider this as a rather technical way to encode the properties: it is quite hard to intuitively understand what such a bisimilarity goal actually entails and what not. While one can get insights from a failed proof when the goal is too strong, one cannot easily see when it is too weak (with respect to one's intuition).

This paper gives a model-theoretic way to formalize and reason about privacy and receipt-freeness. In our extended version we also discuss coercion resistance. We build on the framework of $(\alpha, \beta)$-privacy [5], that defines privacy as a state-based safety property, where each state consists of two formulae $\alpha$, the deliberately released high-level information like the result of an election, and $\beta$, the observations that the intruder could make. During transitions, the information in $\alpha$ and $\beta$ can only increase (by adding new conjuncts to the formulae). The question is (for every reachable state), whether every model of $\alpha$ is compatible with the observations in $\beta$ : if not, the intruder is able to rule out some models of $\alpha$ and thus has obtained more information about the system than we have deliberately released. In particular we use this to come as close as possible to the following intuitive definition of privacy goals:

(a) Voting privacy: the number of voters and the result of the election are finally published. The intruder should not find out more than that about voters and votes.

(b) Receipt-freeness: no voter has a way to prove how they voted. This can be indirectly expressed by saying: for everything that could have happened 
according to (a), the voter can make up a consistent "story".

We do not necessarily regard our definitions as a replacement for existing approaches [6]. We do not present an automated verification procedure, but we see this as complementing the view and giving new ideas to understand the subtlety of goals, and possibly also paving the way for new verification methods. The logical approach allows for declarative manual proofs like reasoning about a permutation of votes and the intruder's knowledge about that permutation. We give examples of proofs for the most interesting states of the simple voting protocol FOO'92 8 to illustrate all the concepts. Proofs can be found in the appendix.

\section{Preliminaries}

It is common in protocol verification to consider an algebraic model of messages, namely interpreting functions in the quotient algebra modulo a set of algebraic equations (i.e., two terms are different unless the algebraic equations deem them equal). Many approaches usually reason about only logical implications, i.e. derivations that follow in every interpretation. In contrast, we here reason about the different interpretations of formulae and hence have to make the interpretation of functions explicit. For this reason, we use Herbrand logic 7], a variant of first-order logic, that allows us just that.

\subsection{Herbrand Logic}

For brevity, we only highlight the differences to standard first-order logic, the precise definition that we use can be found in the original paper on $(\alpha, \beta)$ privacy 5]. The main point is that in Herbrand logic fixes the universe in which to interpret all symbols. To that end, we consider always a signature $\Sigma=\Sigma_{f} \uplus \Sigma_{i} \uplus \Sigma_{r}$ that distinguishes three kinds of symbols: $\Sigma_{f}$ the set of uninterpreted function symbols, $\Sigma_{i}$ the set of interpreted function symbols and $\Sigma_{r}$ the set of relation symbols. Let $\left.\mathcal{T}\right\rfloor_{\Sigma_{f}}$ be the set of (ground) terms that can be built using symbols in $\Sigma_{f}$, and let $\approx$ be a congruence relation on $\left.\mathcal{T}\right\rfloor_{\Sigma_{f}}$, then the Herbrand universe is the quotient algebra $\mathcal{A}\rfloor=\mathcal{T}\rfloor_{\Sigma_{f}} / \approx$. Note that the universe $A$ of $\mathcal{A}\rfloor$ thus consists of equivalence classes of terms, i.e., $\left.A=\{\llbracket t \rrbracket \approx \mid t \in \mathcal{T}\rfloor_{\Sigma_{f}}\right\}$ where $\left.\llbracket t \rrbracket \approx=\left\{t^{\prime} \mid t \in \mathcal{T}\right\rfloor_{\Sigma_{f}} \wedge t \approx t^{\prime}\right\}$. Algebra $\left.\mathcal{A}\right\rfloor$ also fixes the "interpretation" of all uninterpreted function symbols, namely $f^{\mathcal{A}\rfloor}\left(\llbracket t_{1} \rrbracket \approx, \ldots, \llbracket t_{n} \rrbracket \approx\right)=\llbracket f\left(t_{1}, \ldots, t_{n}\right) \rrbracket \approx$.

The interpreted function symbols $\Sigma_{i}$ and the relation symbols $\Sigma_{r}$ behave as in standard first-order logic, namely as function and relation symbols on the universe. To highlight syntactically the distinction between uninterpreted and interpreted function symbols, we write $f\left(t_{1}, \ldots, t_{n}\right)$ if $f \in \Sigma_{f}$ and $f\left[t_{1}, \ldots, t_{n}\right] \in$ $\Sigma_{i}$. Given a signature $\Sigma$, a set of variables $\mathcal{V}$ distinct from $\Sigma$, and a congruence $\approx$, and thus fixing a universe $A$, we define an interpretation $\mathcal{I}$ (w.r.t. $\Sigma, \mathcal{V}$, and $\approx)$ as a function such that $\mathcal{I}(x) \in A$ for every $x \in \mathcal{V}, \mathcal{I}(f): A^{n} \rightarrow A$ for every 
$f \in \Sigma_{i}$ of arity $n$, and $\mathcal{I}(r) \subseteq A^{n}$ for every $r \in \Sigma_{r}$ of arity $n$. (The functions of $\Sigma_{f}$ are determined by the quotient algebra.) We define a model relation $\mathcal{I}=\phi$ (formula $\phi$ holds under $\mathcal{I}$ ) as standard and use common notations like $\phi=\psi$.

For the rest of the paper, we assume that $\Sigma_{f}$ contains the constant 0 and the unary function $s$, and $\Sigma_{i}$ contains the binary function + . This means that the universe contains the natural numbers $0, s(0), s(s(1)), \ldots$ which we often write as $0,1,2, \ldots$. We characterize + by the following axiom that we also generally assume for the rest of this paper:

$$
\alpha_{a x}=\forall x, y . \quad x+0=x \wedge x+s(y)=s(x+y)
$$

Note that this characterization is only possible due to the expressive power of Herbrand logic (in standard First-Order Logic one cannot characterize the universe appropriately).

\subsection{Encoding of frames}

We use, as it is standard in security protocol verification, a black-box algebraic model. We choose a subset $\Sigma_{o p} \subseteq \Sigma_{f}$ of uninterpreted functions to be the operators available to the intruder. For instance we generally require $0, f \in$ $\Sigma_{o p}$, so the intruder can "generate" any natural number 11 For representing the intruder knowledge, we use the concept of frames:

Definitinon 1 (Frame [5]). A frame is written as $\digamma=\left\{m_{1} \mapsto t_{1}, \ldots, m_{l} \mapsto t_{l} \mid\right\}$ where the $m_{i}$ are distinguished constants and the $t_{i}$ are terms that do not contain any $m_{i}$. We call $m_{1}, \ldots, m_{l}$ the domain and $t_{1}, \ldots, t_{l}$ the image of the frame. We write $\digamma\{t\}\}$ for replacing in the term $t$ every occurrence of $m_{i}$ with $t_{i}$, i.e., $\digamma$ works like a substitution. $\digamma^{D}$ denotes the restriction of $\digamma$ to sub-domain $D$.

The $m_{i}$ can be regarded as memory locations of the intruder, representing that the intruder knows the messages $t_{i}$. The set of recipes is the least set that contains $m_{1}, \ldots, m_{l}$ and that is closed under all the cryptographic operators $\Sigma_{o p}$.

In order to formalize frames in Herbrand logic, we introduce two new symbols: a unary predicate symbol gen that characterizes the set of recipes and a unary interpreted function symbol $k n_{\digamma}$ that encodes the frame $k n$ as a function from recipes to messages. The following axioms characterize these symbols.

Definitinon $2\left(\phi_{\text {gen }}(D)\right.$ and $\left.\phi_{\text {frame }}(\digamma)[5]\right)$. For a frame $\digamma=\left\{m_{1} \mapsto t_{1}, \ldots\right.$, $\left.m_{l} \mapsto t_{l} \mid\right\}$ with domain $D=\left\{m_{1}, \ldots, m_{l}\right\}$, a unary predicate gen and an inter-

\footnotetext{
${ }^{1}$ The fact that $+\notin \Sigma_{o p}$ does not limit the intruder: all natural arithmetic reasoning is part of the models relation already (thanks to $\alpha_{a x}$ ).
} 
preted unary function symbol $k n_{\digamma}$, we define the Herbrand logic formulae:

$$
\begin{aligned}
& \phi_{\text {gen }}(D) \equiv \forall r . g e n(r) \Longleftrightarrow \\
& \left(r \in D \vee \bigvee_{f^{n} \in \Sigma_{o p}} \exists r_{1}, \ldots, r_{n} . r=f\left(r_{1}, \ldots, r_{n}\right) \wedge \operatorname{gen}\left(r_{1}\right) \wedge \ldots \wedge \operatorname{gen}\left(r_{n}\right)\right) \\
& \phi_{\text {frame }}(\digamma) \equiv k n_{\digamma}\left[m_{1}\right]=t_{1} \wedge \ldots \wedge k n_{\digamma}\left[m_{l}\right]=t_{l} \wedge \\
& \bigwedge_{f^{n} \in \Sigma_{o p}} \forall r_{1}, \ldots, r_{n}: \text { gen. } k n_{\digamma}\left[f\left(r_{1}, \ldots, r_{n}\right)\right]=f\left(k n_{\digamma}\left[r_{1}\right], \ldots, k n_{\digamma}\left[r_{n}\right]\right)
\end{aligned}
$$

The axiom $\phi_{g e n}(\digamma)$ defines the unary predicate gen to be the set of recipes from $D$ and $\Sigma_{o p}$. Here we write $f^{n} \in \Sigma_{o p}$ to denote that $f$ is an $n$-ary function symbol. We can use gen like a type and for instance write $\forall r:$ gen. $\phi$ as shorthand for $\forall r . g e n(r) \Longrightarrow \phi$. The axiom $\phi_{\text {frame }}(\digamma)$ characterizes that $k n_{\digamma}$ works like a substitution on recipes, mapping $m_{i}$ to $t_{i}$.

Two frames $\digamma_{1}$ and $\digamma_{2}$ with the same domain are called statically equivalent, if on every pair of recipes, $\digamma_{1}$ produces the same result iff $\digamma_{2}$ does:

Definitinon $3\left(\phi_{\sim}\left(\digamma_{1}, \digamma_{2}\right)[5]\right)$. Let $\digamma_{1}$ and $\digamma_{2}$ be frames with the same domain.

$$
\phi_{\sim}\left(\digamma_{1}, \digamma_{2}\right) \equiv \forall r, s: \text { gen. } k n_{\digamma_{1}}[r]=k n_{\digamma_{1}}[s] \Longleftrightarrow k n_{\digamma_{2}}[r]=k n_{\digamma_{2}}[s]
$$

\subsection{Alpha-Beta Privacy}

$(\alpha, \beta)$-privacy is mainly based on the specification of two formulae $\alpha$ and $\beta$ in Herbrand Logic for every reachable state. $\alpha$ is the intentionally released information, i.e. the "non-technical" information or the "payload". $\beta$ represents all other observations that the intruder made, e.g. messages he has seen, also called the "technical" information. This distinction between payload and technical is at the core of $(\alpha, \beta)$-privacy. We formalize it by a distinguished subset $\Sigma_{0} \subset \Sigma$ of the alphabet, where $\Sigma_{0}$ contains only the non-technical information, such as votes and addition, while $\Sigma \backslash \Sigma_{0}$ includes cryptographic operators. The formula $\alpha$ is always over just $\Sigma_{0}$, while $\beta$ can use the full $\Sigma$. $(\alpha, \beta)$-privacy from 5 :

Definitinon 4 (Model-theoretical $(\alpha, \beta)$-privacy [5]). Consider a countable signature $\Sigma$ and a payload alphabet $\Sigma_{0} \subset \Sigma$, a formula $\alpha$ over $\Sigma_{0}$ and a formula $\beta$ over $\Sigma$ such that $f v(\alpha)=f v(\beta)$, both $\alpha$ and $\beta$ are consistent and $\beta=\alpha$. We say that $(\alpha, \beta)$-privacy holds (model-theoretically) iff every $\Sigma_{0}$-model of $\alpha$ can be extended to a $\Sigma$-model of $\beta$. Here a $\Sigma$-interpretation $\mathcal{I}^{\prime}$ is an extension of a $\Sigma_{0}$-interpretation $\mathcal{I}$ if they agree on all variables and all the interpreted function and relation symbols of $\Sigma_{0}$.

For the rest of this paper, we consider as $\alpha$ only combinatoric formulae, which means $\Sigma_{0}$ is finite and contains only uninterpreted constants. Then $\alpha$ has only finitely many models (that assign constants of $\Sigma_{0}$ to the free variables of $\alpha$ ). 
While classical bi-similarity approaches are always about the distinguishability between two alternatives, in $(\alpha, \beta)$-privacy every reachable state represents only one single situation that can occur, and it is the question how far the intruder can know what happened. The intruder knowledge is for this reason more complex: besides the concrete messages he knows, we also model that he may know something about the structure of messages, e.g. that a particular encrypted message in his knowledge contains the vote $v_{1}$-where $v_{1}$ is a free variable of $\alpha$. We thus define the intruder knowledge by two frames concr and struct where struct may contain free variables of $\alpha$, and concr is the same except that all variables are instantiated with what really happened, e.g. $v_{1}=1$. For simplicity let us use in Herbrand formulae also concr and struct as two interpreted functions (instead of $k n_{\text {concr }}$ and $k n_{\text {struct }}$ ) and we call them the concrete and structural knowledge and we always use gen to refer to the recipes for both (since they must have the same domain). The clou is that we require as part of $\beta$ that struct and concr are statically equivalent. That means, if the intruder knows that two concrete constructible messages are unequal, then also their structure has to be unequal, and vice-versa. For instance, let $h \in \Sigma_{p} \backslash \Sigma_{o p}$ and struct $=\left\{m_{1} \mapsto h\left(v_{1}\right), m_{2} \mapsto h\left(v_{2}\right)\right\}$ and concr $=\left\{m_{1} \mapsto h(0), m_{2} \mapsto h(1)\right\}$. Then every model of $\beta$ has the property $v_{1} \neq v_{2}$. Suppose $\alpha \equiv v_{1}, v_{2} \in\{0,1\}$, then $(\alpha, \beta)$-privacy is violated, since for instance $v_{1}=0, v_{2}=0$ is a model of $\alpha$, but cannot be extended to a model of $\beta$. If $\alpha \equiv v_{1}, v_{2} \in\{0,1\} \wedge v_{1}+v_{2}=1$ however, then all models of $\alpha$ are compatible with $\beta$ and privacy is preserved.

Definitinon 5 (Message-analysis problem (adapted from [5])). Let $\alpha$ be combinatoric, struct and concr be two frames with domain $D$. We say that $\beta$ is a message-analysis problem if $\beta \equiv \operatorname{MsgAna}(D, \alpha$, struct, concr $)$ with:

$\operatorname{MsgAna}(D, \alpha$, struct, concr $) \equiv \alpha \wedge \phi_{\text {gen }}(D) \wedge \phi_{\text {frame }}($ struct $) \wedge \phi_{\text {frame }}($ concr $) \wedge \phi_{\sim}($ struct, concr $)$

Typically, we consider for every state one distinguished model $\theta$ of $\alpha$, called the reality, and have concr $=\theta$ (struct).

\section{$3 \quad$ Verifying Voting Privacy}

An $(\alpha, \beta)$-pair characterizes a single state of a transition system. To illustrate voting privacy and receipt-freeness we pick a few reachable states of the voting protocol FOO'92 and prove (or disprove) fulfillment of some properties. In fact, a manual proof for the entire infinite state-space is possible by appropriate generalization, but for the purpose of illustration, this would be overkill.

First, let us look at $\alpha$ and for simplicity consider a choice between 0 or 1 (all definitions can be easily extended to more complex voting choices). We use a sequence of variables $v_{1}, \ldots, v_{N}$ to model the votes. During each transition where an honest voter $i$ sends a message that contains their vote $v_{i}$, we augment $\alpha$ by $v_{i} \in\{0,1\}$, since the intruder does not know more than they will cast a valid vote. For dishonest voters, it is more complicated and actually depends on the protocol, since dishonest voters (or the intruder) may not follow the 
protocol and, e.g., replay a message of some honest voter (thus not necessarily knowing what vote they have cast). Anyway in this case one should augment $\alpha$ with $v_{i}=b$ for the concrete $b$ that they have cast, since the intruder is allowed to know all dishonest votes. Finally, when the result is about to be published, let us say $R$ votes $v_{i}$ are 1 , then we finally augment $\alpha$ with the information $\sum_{i=1}^{N} v_{i}=R$. Thus, from this point on the intruder is allowed to know the result, but until then, it is even a violation if he can obtain a partial result (beyond the votes of the dishonest agents). For all examples in this paper we have

$$
\alpha \equiv v_{1} \in\{0,1\} \wedge \ldots \wedge v_{N} \in\{0,1\} \wedge \sum_{i=1}^{N} v_{i}=R
$$

i.e., $N$ honest voters have cast their votes, and $R$ of them are 1 . In fact, we also use the same formula in examples for receipt-freeness since we want that the same amount of information is kept secret, just under more pressure on the honest voters.

\subsection{The FOO'92 Voting Protocol in Alpha-Beta Privacy}

The FOO'92 protocol 8 has been formally studied with the Applied $\pi$-calculus 9 ; for a full description we refer to that paper, and we only introduce relevant aspects on the fly. The final result of FOO'92 is the publication of a bulletin board of cryptographic messages containing all the votes. More precisely, each entry contains verify $\left(\operatorname{priv}(A), \operatorname{commit}\left(v_{i}, r_{i}\right)\right)$ and $r_{i}$ for some $i \in\{1, \ldots, N\}$. This is the signature with the private key of an administrator $A$ and containing a cryptographic commitment of the vote with some (initially secret) random value $r_{i}$. Here we assume as part of $\Sigma_{o p}$ the following operators: verify for signature, verify for signature verification, and retrieve for obtaining the message under the signature; this is characterized by the equations retrieve $(\operatorname{verify}(\operatorname{priv}(A), m)) \approx m$ and verify $(\operatorname{pub}(A), \operatorname{verify}(\operatorname{priv}(A), m)) \approx y e s$. Moreover we have commit, vcommit and open for commitments with the properties open $(\operatorname{commit}(m, r), r) \approx m$ and $v \operatorname{commit}(r, \operatorname{commit}(m, r)) \approx$ yes.

Let us consider an intruder who just obtains this bulletin board. This is not unrealistic since the exchanges in the other phases are best protected by anonymous channels, anyway. It is crucial that the bulletin board lists its entries in some unpredictable order. To model that, we introduce an interpreted function $\pi[\cdot]$ that is a permutation on $\left.\{1, \ldots, N\}\right|^{2}$ To conveniently make use of this function, we like to also access the votes $v_{i}$ and the random values $r_{i}$ (these are uninterpreted constants of $\left.\Sigma \backslash \Sigma_{0}\right)$ through a function and thus introduce two further interpreted functions $v[\cdot]$ and $r[\cdot]$ with the property $v_{i}=v[i]$ and $r_{i}=r[i]$ for each $1 \leq i \leq N$.

We can then describe the structural knowledge of the intruder who initially

${ }^{2}$ i.e. $\forall i .1 \leq i \leq N \Longrightarrow 1 \leq \pi[i] \leq N \wedge \forall j .1 \leq j \leq N \wedge \pi[i]=\pi[j] \Longrightarrow i=j$. 
knows all public keys and has seen the bulletin board by the following frame:

$$
\begin{aligned}
\text { struct }=\left\{m_{0} \mapsto \operatorname{pub}(A), m_{1} \mapsto \operatorname{pub}\left(V_{1}\right), \ldots, m_{n} \mapsto \operatorname{pub}\left(V_{N}\right),\right. \\
m_{N+1} \mapsto \operatorname{verify}(\operatorname{priv}(A), \operatorname{commit}(v[\pi[1]], r[\pi[1]])), \ldots, \\
m_{2 N} \mapsto \operatorname{verify}(\operatorname{priv}(A), \operatorname{commit}(v[\pi[N]], r[\pi[N]])), \\
\left.m_{2 N+1} \mapsto r[\pi[1]], \ldots, m_{3 N} \mapsto r[\pi[N]]\right\}
\end{aligned}
$$

The concrete knowledge is obtained by instantiating $\pi$ and $v_{i}$ with the actual values. For $\pi$ this means the actual permutation on the bulletin board; let us call it $\pi_{0}$, note this is not a function symbol of the logic but an actual permutation. Further let $\theta_{0}=\alpha$ be an interpretation of the $v_{i}$ with 0,1 that is a model of $\alpha$, i.e., the true vote of every voter. Note that both $\pi_{0}$ and $\theta_{0}$ are arbitrary, so the proofs we give hold for every such choice. Now we can specify concr as follows:

$$
\begin{aligned}
& \text { concr }=\left\{m_{0} \mapsto \operatorname{pub}(A), m_{1} \mapsto \operatorname{pub}\left(V_{1}\right), \ldots, m_{n} \mapsto \operatorname{pub}\left(V_{N}\right),\right. \\
& m_{N+1} \mapsto \operatorname{verify}\left(\operatorname{priv}(A), \operatorname{commit}\left(\theta_{0}\left(v_{\pi_{0}(1)}\right), r_{\pi_{0}(1)}\right)\right), \ldots, \\
& m_{2 N} \mapsto \operatorname{verify}\left(\operatorname{priv}(A), \operatorname{commit}\left(\theta_{0}\left(v_{\pi_{0}(N)}\right), r_{\pi_{0}(N)}\right)\right), \\
& \left.m_{2 N+1} \mapsto r_{\pi_{0}(1)}, \ldots, m_{3 N} \mapsto r_{\pi_{0}(N)}\right\}
\end{aligned}
$$

Thus the concr frame replaces every occurrence of $v[\pi[i]]$ by $\theta_{0}\left(v_{\pi_{0}(i)}\right)$ and every $r[\pi[i]]$ by $r_{\pi_{0}(i)}$. The point is that in the concrete messages that the intruder observes, everything is instantiated with respect to $\pi_{0}$ and $\theta_{0}$, while the structural knowledge about these messages is with respect to $\pi[\cdot]$ and $v[\cdot]$, i.e., reflecting what the intruder knows that about the content of a message, e.g. $v[\pi[j]]$ reflects that the intruder knows that this is the vote of the voter who has entry number $j$ on the bulletin board, but he may be unable to find out the true permutation $\pi_{0}$ and the votes directly as a function of the voters. Using Definition 5, with domain $D=\left\{m_{0}, \cdots, m_{3 N}\right\}$, we define the formula $\beta$ for the scenario that the intruder just sees the bulletin board as follows:

$$
\beta \equiv \bigwedge_{i=1}^{N}\left(v[i]=v_{i} \wedge r[i]=r_{i}\right) \wedge \operatorname{MsgAna}(D, \alpha, \text { struct }, \text { concr })
$$

Let us call $S$ the state with this $\beta$ and the $\alpha$ of (1).

\subsection{Voting privacy holds in $\mathrm{S}$}

To show that $(\alpha, \beta)$-privacy holds in $S$, we need to show how an arbitrary model of $\alpha$ can be extended to a model of $\beta$. To that end, we consider an arbitrary model $\theta_{I}=\alpha$, called an intruder hypothesis, i.e., that maps the $v_{i}$ to $\{0,1\}$ so that their sum is $R$. We show how $\theta_{I}$ it can be extended to a model $\mathcal{I} \models \beta$. In other words, we show that $\beta$ does not allow the intruder to logically rule out any hypothesis about the votes $v_{i}$. We do this construction for an arbitrary $\theta_{I}$, thus, every model of $\alpha$ can be extended to a model of $\beta$.

Since $\mathcal{I}$ must be an extension of $\theta_{I}$, we have $\mathcal{I}\left(v_{i}\right)=\theta_{I}\left(v_{i}\right)$ for all votes $v_{i}$. Further, we need to give an interpretation for all other symbols of $\Sigma$, in our 
example $\operatorname{gen}(\cdot)$, struct $[\cdot]$, concr $[\cdot], \pi[\cdot], r[\cdot]$ and of course $v[\cdot]$. For the symbols gen, struct, and concr there is not much choice so that they satisfy the formulae $\phi_{\text {gen }}, \phi_{\text {frame }}\left(\right.$ struct) and $\phi_{\text {frame }}($ concr $)$, and we give a canonical construction for them (i.e., the same construction applies for any message analysis problem). More interesting is to find an interpretation of the protocol specific functions $\pi[\cdot]$, $r[\cdot]$ and $v[\cdot]$, so that $\mathcal{I}=\phi_{\sim}($ struct, concr $)$, i.e., verifying the static equivalence struct and concr modulo $\approx$. While this is generally difficult, we are sometimes in luck: in many cases $(\alpha, \beta)$-privacy allows for a relatively easy proof by reasoning about permutations - i.e. how "human provers" would like to do it. Indeed, for the state $S$, we can find an interpretation for $\pi[\cdot]$ (and the other functions) such that $\mathcal{I}($ struct $)=\mathcal{I}($ concr $)$. In this case $\mathcal{I} \models \phi_{\sim}($ struct, concr $)$ follows trivially. Note that here, we do not even need to reason about algebraic properties of the operators (i.e. the congruence relation $\approx$ ) to conduct the proof.

The proof idea for this is actually quite straightforward in this case. Remember that $S$ entails "what really happened", i.e., a particular model $\theta_{0} \models \alpha$ and a particular permutation $\pi_{0}$ that reflect the true outcome of the vote, and the true permutation under which the votes have been published. The idea is that any discrepancy between $\theta_{I}$ and $\theta_{0}$ can be "balanced" by an appropriate interpretation of $\pi$. More precisely, the voting function is interpreted following the intruder hypothesis, i.e. $v[i]$ is $\theta_{I}\left(v_{i}\right)$ for all voters. Since both $\theta_{0} \models \alpha$ and $\theta_{I} \models \alpha$, we have $\sum_{i=1}^{N} \theta_{0}\left(v_{i}\right)=\sum_{i=1}^{N} \theta_{I}\left(v_{i}\right)=R$. Since $v_{1}, \ldots, v_{N} \in\{0,1\}$, the list $\left[\theta_{I}\left(v_{1}\right), \ldots, \theta_{I}\left(v_{N}\right)\right]$ is a permutation of $\left[\theta_{0}\left(v_{1}\right), \ldots, \theta_{0}\left(v_{N}\right)\right]$. Thus we can find a permutation $\psi:\{1, \ldots, N\} \rightarrow\{1, \ldots, N\}$ such that $\theta_{I}\left(v_{i}\right)=\theta_{0}\left(v_{\psi(i)}\right)$ for all $i \in\{1, \ldots, N\}$. Intuitively, $\psi$ is the discrepancy between $\theta_{I}$ and $\theta_{0}$. Then let us define $\pi_{I}$ as the intruder's hypothesis of $\pi: \pi_{I}=\psi^{-1} \circ \pi_{0}$. Finally, $r$ is interpreted accordingly, as the commitment secrets permuted the same way that the votes, i.e. a value $r[i]$ is $r_{\psi(i)}$. Let us thus define (recall the Herbrand universe is $\left.\left.A=\{\llbracket t \rrbracket \approx \mid t \in \mathcal{T}\rfloor_{\Sigma_{f}}\right\}\right)$ :

Definitinon 6 (A model of the functions). Let $\mathcal{I}$ map $v$ to the function $\mathcal{I}(v): A \rightarrow$ $A, r$ to the function $\mathcal{I}(r): A \rightarrow A$ and $\pi$ to the function $\mathcal{I}(\pi): A \rightarrow A$ :

$$
\begin{array}{lll}
\mathcal{I}(v)(\llbracket t \rrbracket \approx)=\llbracket \theta_{I}\left(v_{t}\right) \rrbracket \approx & \text { if } t \in \llbracket\{1, \ldots, N\} \rrbracket \approx \text { and } \mathcal{I}(v)(\llbracket t \rrbracket \approx)=\llbracket t \rrbracket \approx \text { otherwise } \\
\left.\mathcal{I}(r)(\llbracket t \rrbracket \approx)=\llbracket r_{\psi}\right)= & \text { if } t \in \llbracket\{1, \ldots, N\} \rrbracket \approx \text { and } \mathcal{I}(r)\left(\llbracket t \rrbracket_{\approx}\right)=\llbracket t \rrbracket \approx \text { otherwise } \\
\mathcal{I}(\pi)(\llbracket t \rrbracket \approx)=\llbracket r_{I}(t) \rrbracket \approx & \text { if } t \in \llbracket\{1, \ldots, N\} \rrbracket \approx \text { and } \mathcal{I}(\pi)(\llbracket t \rrbracket \approx)=\llbracket t \rrbracket \approx \text { otherwise }
\end{array}
$$

The construction of the remaining items is generic for all message analysis problems, namely struct and concr behave like substitutions and that gen is true exactly for the recipes:

Definitinon 7 (A model of gen, struct and concr). Let $D$ be the domain of the considered frames. Then we define:

$$
\begin{aligned}
\mathcal{I}(\text { gen }) & =\left\{\llbracket t \rrbracket \approx \mid t \in \mathcal{T}_{\Sigma_{o p} \cup D}\right\} \\
\mathcal{I}(\text { struct })(\llbracket t \rrbracket \approx) & =\mathcal{I}(\text { struct }\{t\}) \text { for all } t \in \mathcal{T}_{\Sigma_{f}} \\
\mathcal{I}(\text { concr })(\llbracket t \rrbracket \approx) & =\mathcal{I}(\text { concr }\{\mid t\}) \text { for all } t \in \mathcal{T}\rfloor_{\Sigma_{f}}
\end{aligned}
$$


This interpretation expresses that gen is exactly the set of recipes. For struct and concr, we define the meaning by first applying the actual frames struct $\{|\cdot|\}$ and $\operatorname{concr}\{|\cdot|\}$ as substitutions to a given term $t$, i.e. replacing the labels $m_{i} \in D$ in $t$; afterwards, we apply $\mathcal{I}$ to the resulting term since struct $\{t \mid\}$ in general contains variables and interpreted function symbols that need to be interpreted. This interpretation is well-defined because it does not depend on the choice of the representative of the equivalence classes, e.g. if $s \approx t$ then struct $\{|s|\} \approx \operatorname{struct}\{t \mid\}$. It is immediate that $\mathcal{I}$ is a model of $\phi_{\text {frame }}($ struct) and $\phi_{\text {frame }}($ concr $)$ :

Lemma 1. $\mathcal{I} \models \phi_{\text {frame }}($ struct $)$ and $\mathcal{I} \models \phi_{\text {frame }}($ concr $)$.

It remains to show that $\mathcal{I}=\phi_{\sim}($ struct, concr $)$. In general, such a proof of static equivalence of two frames can be difficult (especially by hand). However, in our case we have $\mathcal{I}$ (struct) $=\mathcal{I}($ concr $)$ by construction - we have designed the interpretation of $\pi$ so that this holds - and then static equivalence is immediate:

Lemma 2. If $\mathcal{I}($ struct $)=\mathcal{I}($ concr $)$ then $\mathcal{I} \models \phi_{\sim}($ struct, concr $)$.

Theorem 1. Voting privacy holds in the state $S$.

This FOO'92 example demonstrates the declarativity of the $(\alpha, \beta)$-privacy approach, in particular that we are able to reason about permutations allows for a rather simple proof how "human provers" would like it: after a small insight (the discrepancy between $\theta_{I}$ and $\theta_{0}$ can be balanced in the interpretation of $\pi$ ) then the rest all falls into place.

\subsection{Voting privacy holds in S'}

In many cases it is not as easy as before. For instance, in the FOO'92 protocol, we have a first phase where send a blind signature of their vote-commitment to an administrator and receive a signature from that administrator. Let us now consider a state $S^{\prime}$ where the intruder has seen also all theses blinded signatures (the formula $\alpha$ is again (1)):

$$
\begin{aligned}
& \operatorname{struct}=\left\{m_{0} \mapsto \operatorname{pub}(A), m_{1} \mapsto \operatorname{pub}\left(V_{1}\right), \ldots, m_{n} \mapsto \operatorname{pub}\left(V_{N}\right),\right. \\
& m_{N+1} \mapsto \operatorname{verify}(\operatorname{priv}(A), \operatorname{commit}(v[\pi[1]], r[\pi[1]])), \ldots, \\
& m_{2 N} \mapsto \operatorname{verify}(\operatorname{priv}(A), \operatorname{commit}(v[\pi[N]], r[\pi[N]])), \\
& m_{2 N+1} \mapsto r[\pi[1]], \ldots, m_{3 N} \mapsto r[\pi[N]], \\
& m_{3 N+1} \mapsto \operatorname{verify}\left(\operatorname{priv}\left(V_{1}\right), \operatorname{blind}\left(\operatorname{commit}(v[1], r[1]), b_{1}\right)\right), \ldots, \\
& m_{4 N} \mapsto \operatorname{verify}\left(\operatorname{priv}\left(V_{N}\right), \operatorname{blind}\left(\operatorname{commit}(v[N], r[N]), b_{N}\right)\right), \\
& m_{4 N+1} \mapsto \operatorname{verify}\left(\operatorname{priv}(A), \operatorname{blind}\left(\operatorname{commit}(v[1], r[1]), b_{1}\right)\right), \ldots, \\
& \left.\left.m_{5 N} \mapsto \operatorname{verify}\left(\operatorname{priv}(A), \operatorname{blind}\left(\operatorname{commit}(v[N], r[N]), b_{N}\right)\right)\right\}\right\}
\end{aligned}
$$

Here, we have augmented the frame from $S$ by the $3 N+i$ messages from the voters and the $4 N+i$ replies from the administrator, and $b_{i}$ is the corresponding 
blinding secret of voter $V_{i}$. We assume the following properties about blind: $\operatorname{blind}(x, \operatorname{blind}(x, m)) \approx m$ and $\operatorname{verify}(x, \operatorname{blind}(y, m)) \approx \operatorname{blind}(y, \operatorname{verify}(x, m))$, so that each voter can obtain the unblinded the reply the message from the administrator. The concrete frame concr is again obtained by replacing $\pi[x]$ by $\pi_{0}(x), v[x]$ by $\theta_{0}\left(v_{x}\right)$ and $r[x]$ by $r_{x}$.

Note that the messages between voters and administrators are actually shown in the order of the voters rather than under a permutation. The reason is that such a permutation would not make the problem harder for the intruder, since the signatures of the voters already identify which message belongs to whom and the replies from the administrator could probably be linked due to timing.

Now the difficulty is that we cannot find an interpretation $\mathcal{I}$ such that $\mathcal{I}($ struct $)=\mathcal{I}($ concr $)$, because the messages at $3 N+i$ are signed by the individual voters and are thus linked to the voters ${ }^{3}$ Instead, the point is here that, due to the blinding, the intruder cannot derive anything useful from these messages. Formally, we show for the same $\mathcal{I}$ as constructed for $S$ (for given $\left.\theta_{I} \models \alpha\right)$, that the weaker property $\mathcal{I} \models \phi_{\sim}($ struct, concr $)$ still holds in $S^{\prime}$. This therefore requires a full static equivalence proof modulo the properties of $\approx$ which is quite involved (cf. for instance [13]) and we give only a sketch in the appendix. This allows us to conclude:

Theorem 2. Voting privacy holds in the state $S^{\prime}$.

\section{Receipt-freeness}

We now assume that the intruder tries to influence one particular voter, let us call him Dan and identify him with voter $1 V_{1}$. We will later briefly discuss the case when then intruder tries to influence several voters. The question is whether Dan can prove to the intruder how he voted by a kind of "receipt" how he voted. The protocol does not explicitly produce any such receipt, but revealing all messages that Dan knows could allow the intruder to verify how Dan voted, i.e., that Dan is unable to lie about his vote. For instance, in the case of FOO'92, if the intruder has observed all the messages between voters and administrators, and if Dan reveals his blinding factor, then the intruder can indeed identify Dan's vote with certainty. If we consider however FOO'92 without the blinded signature messages (as in state $S$ ) and the intruder sees only the final bulletin board, Dan can claim any vote to be his - and the intruder has no chance to falsify that claim. Mind that does not hold in the state before the commitments are opened as we also discuss below.

\footnotetext{
${ }^{3}$ In fact, due to the $3 N+i$ messages, in any model $\mathcal{I}$ where $\mathcal{I}$ (struct) $=\mathcal{I}$ (concr) we necessarily also have $\theta_{0}=\theta_{I}$, and thus there cannot be this simple construction for every $\theta_{I} \models \alpha$.
} 


\subsection{Formalizing receipt-freeness}

Consider a given state where we want to check whether the protocol is receiptfree with respect to the voter Dan. The intruder can ask Dan to reveal his entire knowledge, i.e., all the secrets Dan knows (his private key, his commitment value and his blinding factor) as well as messages that Dan has received from other parties, like the administrator. If Dan has any "receipt" (in the broadest sense of the word), then it is something that can be derived from this knowledge. The point is that Dan does not necessarily tell the truth, but can present any collection of messages that can be constructed from his knowledge. We call this Dan's story. Dan's story has to be consistent with whatever the intruder can check, e.g., Dan cannot lie about his private key, since the intruder knows his public key. We thus want to express that a state is receipt-free, if for every model $\theta_{I} \models \alpha$, Dan can come up with a consistent story (in particular consistent with $\left.\theta_{I}\right)$. We do not even change the formula $\alpha$, but only add an additional challenge to $\beta$ : that the intruder obtains a story from Dan, i.e. what he claims to be his knowledge. We see receipt-freeness as preserving voting privacy even under this additional challenge. From that actually follows a relation between the goals: receipt freeness implies voting privacy.

We reason about Dan's knowledge similarly to the intruder's knowledge: we

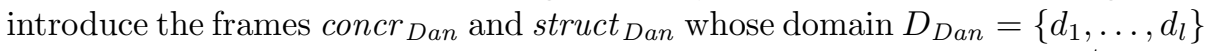
is disjoint from the domain $D$ of the intruder knowledge: $D_{\operatorname{Dan}} \cap D=\emptyset$. If we consider that the protocol itself is not a secret, the intruder "knows" struct ${ }_{\text {Dan }}$, i.e., what the messages are supposed to be according to protocol, and Dan's story has to be consistent with this. The idea is that what Dan can lie about is concr $_{\text {Dan }}$. We let Dan choose any recipes $s_{1}, \ldots, s_{l}$ (with respect to $D_{\text {Dan }}$ ), one for each item in his knowledge and send concr $\left[s_{1}\right], \ldots, \operatorname{concr}\left[s_{l}\right]$ as his story to the intruder. The augmented intruder knowledge has then domain $D \cup D_{\text {Dan }}$ where the concr's are filled with Dan's story and the struct is identical with struct $_{D a n}$, i.e., what it is supposed to be. This is captured by the formula $\phi_{l i e}$ :

Definitinon $8\left(\phi_{\text {lie }}\right)$.

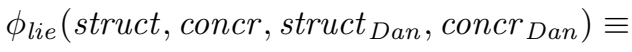

$$
\begin{aligned}
& \text { struct }_{\left[d_{1}\right]}=\text { struct }_{\text {Dan }}\left[d_{1}\right] \wedge \ldots \wedge \operatorname{struct}\left[d_{l}\right]=\text { struct }_{\text {Dan }}\left[d_{l}\right] \\
& \wedge \exists s_{1}, \ldots, s_{l}: \operatorname{gen}_{D_{D a n}} \cdot\left(\operatorname{concr}\left[d_{1}\right]=\operatorname{concr}_{\operatorname{Dan}}\left[s_{1}\right] \wedge \ldots \wedge \operatorname{concr}\left[d_{l}\right]=\operatorname{concr}_{\text {Dan }}\left[s_{l}\right]\right)
\end{aligned}
$$

In fact, $\mathcal{I}=\phi_{\text {lie }}\left(\right.$ concr, struct) (w.r.t. the whole domain $D \cup D_{\text {Dan }}$ ) means that Dan's story is consistent with the protocol (i.e., the struct values) and $\mathcal{I}$ 's interpretation of the free variables of $\alpha$. Thus we define:

Definitinon 9 (Receipt-freeness problem). We say that $\beta$ is a receipt-freeness problem (with respect to a combinatoric $\alpha$, the frames struct and concr with domain $D \cup D_{\text {Dan }}$, the frames struct Dan and concr $_{\text {Dan }}$ with domain $D_{\text {Dan }}$ ) if 
$\beta \equiv \operatorname{RcpFree}\left(D, D_{\text {Dan }}, \alpha\right.$, struct, concr struct $_{\text {Dan }}$, concr $\left._{\text {Dan }}\right)$ where:

$\operatorname{RcpFree}\left(D, D_{\text {Dan }}, \alpha\right.$, struct, concr struct $_{\text {Dan }}$, concr $\left._{\text {Dan }}\right)$

$\equiv \phi_{\text {gen }_{D_{\text {Dan }}}}\left(D_{\text {Dan }}\right) \wedge \phi_{\text {frame }}\left(\right.$ struct $\left._{\text {Dan }}\right) \wedge \phi_{\text {frame }}\left(\right.$ concr $\left._{\text {Dan }}\right)$

$\wedge \operatorname{MsgAna}\left(D \cup D_{\text {Dan }}, \alpha\right.$, struct, concr $) \wedge \phi_{\text {lie }}\left({\left.\text { struct }, \text { concr }, \text { struct }_{\text {Dan }}, \text { concr }_{\text {Dan }}\right)}\right.$

We say receipt-freeness holds if the $(\alpha, \beta)$-pair is consistent. We call $\beta^{\prime} \equiv$ $\alpha \wedge \phi_{\text {gen }}(D) \wedge \phi_{\text {frame }}($ struct $) \wedge \phi_{\text {frame }}($ concr $) \equiv \operatorname{MsgAna}(D, \alpha$, struct, concr $)$ the message-analysis problem underlying $\beta$.

$\beta$ is always consistent since there is at least one way to satisfy $\beta$ : the truth (i.e. Dan selects $s_{i}=d_{i}$ for each $1 \leq i \leq l$ ). The truth may be only compatible with a subset of the models of $\alpha$, but that includes at least one model: $\theta_{0}$, the reality. Note that the receipt-freeness problem is well-suited to model any privacy problem where we want to protect the privacy of a choice from the influence of an intruder.

We relate this formal definition to the informal definition that we gave in the introduction. The consistent "story" is here represented by the axiom $\phi_{\text {lie }}$. For every receipt-freeness problem, we also defined an underlying message-analysis problem that is just a restriction of the original receipt-freeness problem. Indeed, we consider only $\alpha$, the formula relating to the knowledge of the intruder and we restrict the formula for generating recipes to domain $D$. In that sense, we can use the next proposition to build a relation between the privacy properties. It is sufficient to prove receipt-freeness to prove plain voting privacy.

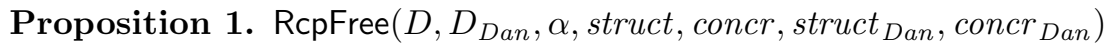
$\models \operatorname{Msg} \operatorname{Ana}(D, \alpha$, struct, concr $)$

\subsection{Receipt-freeness in $\mathrm{S}$}

FOO'92 does not satisfy receipt-freeness as shown in [6], and even though our notion of receipt-freeness is defined differently, it agrees with their results. FOO'92 serves well anyway for illustration: in the final state $S$ that we have considered before (where the intruder has seen only the final bulletin board), receiptfreeness does hold as we now show.

Let us first consider $S$, augment it to a receipt-freeness problem with respect to a voter Dan and show that receipt-freeness actually holds in this state. We first need to define what the knowledge of a voter Dan is. The structural information is very similar to the intruder's information that consists of the published information (the bulletin board and the public keys); additionally Dan also knows his private key, his own vote, his own commitment value and his blinding factor. We did not include the blinded message as they can be 
reconstructed from the blinding factor.

$$
\begin{aligned}
& \text { struct }_{\text {Dan }}=\left\{d_{0} \mapsto \operatorname{pub}(A), d_{1} \mapsto \operatorname{pub}\left(V_{1}\right), \ldots, d_{n} \mapsto \operatorname{pub}\left(V_{N}\right),\right. \\
& d_{N+1} \mapsto \operatorname{verify}(\operatorname{priv}(A), \operatorname{commit}(v[\pi[1]], r[\pi[1]])), \ldots, \\
& d_{2 N} \mapsto \operatorname{verify}(\operatorname{priv}(A), \operatorname{commit}(v[\pi[N]], r[\pi[N]])), \\
& d_{2 N+1} \mapsto r[\pi[1]], \ldots, d_{3 N} \mapsto r[\pi[N]], d_{3 N+1} \mapsto \operatorname{priv}(\operatorname{Dan}), d_{3 N+2} \mapsto v[1], \\
& \left.d_{3 N+3} \mapsto r[1], d_{3 N+4} \mapsto b_{1}\right\}
\end{aligned}
$$

The concrete frame is again obtained by replacing $\pi[x]$ by $\pi_{0}(x), v[x]$ by $\theta_{0}\left(v_{x}\right)$ and $r[x]$ by $r_{x}$. The formula $\alpha$ is the same for receipt-freeness as for voting privacy, i.e., the intruder still not supposed to find out anything more than the published result of the election (in particular not what Dan has voted). He has more information in $\beta$ due to the story that Dan gives to the intruder as part of the receipt-freeness definition:

$$
\beta_{\mathrm{RF}} \equiv \bigwedge_{i=1}^{N} v[i]=v_{i} \wedge r[i]=r_{i} \wedge \operatorname{RcpFree}\left(D, D_{\text {Dan }}, \alpha,{\text { struct } \left., \text { concr }, \text { struct }_{\text {Dan }}, \text { concr }_{\text {Dan }}\right)}\right.
$$

When it comes to crafting his story for the public values, Dan has no choice but to tell the truth. As the intruder knows Dan's public key, Dan also has to tell the truth for his private key. For his blinding factor, he may also use the truth as the intruder has not witnessed the exchange with the administrator. For $d_{3 N+2}$ (the actual vote) and $d_{3 N+3}$ (the commitment value), Dan needs to adapt his story to what the intruder "wants to hear", i.e. to a given $\theta_{I}$ (and $\pi_{I}$ ). Observe at this point the order of quantification here: we want to show that every model $\theta_{I} \models \alpha$ can be extended to a model $\mathcal{I} \models \beta_{\mathrm{RF}}$ where $\beta_{\mathrm{RF}}$ entails an existential quantifier for Dan's story. So we have to show how, given $\theta_{I}$, we can construct $\mathcal{I}$ and a value for the recipes of the story $s_{1}, \ldots, s_{l}$ that satisfies all conditions. We take exactly the same construction for $\mathcal{I}$ (depending on $\theta_{I}$ ) that we used for state $S$, i.e., using the discrepancy $\psi$ between the intruder hypothesis $\theta_{I}$ and the reality $\theta_{0}$ (i.e., such that $\left.\theta_{0}\left(v_{\psi(i)}\right)=\theta_{I}\left(v_{i}\right)\right)$ for interpreting $\pi[\cdot]$, namely as the permutation $\pi_{I}=\psi^{-1} \cdot \pi_{0}$. It is sufficient to show that Dan can make his story consistent with this interpretation, namely by pointing to the vote $\psi(1)$ as being his own vote. Let $d_{N+\psi(1)}$ and $d_{2 N+\psi(1)}$ be the indices in Dan's knowledge for the signed commitment and commitment values on the bulletin board at position $\psi(1)$. He can claim this entry by choosing:

$$
s_{3 N+2}=\operatorname{open}\left(\text { retrieve }\left(d_{N+\psi(1)}\right), d_{2 N+\psi(1)}\right) \text { and } s_{3 N+3}=d_{2 N+\psi(1)}
$$

With this we can conclude:

Theorem 3. $\left(\alpha, \beta_{i \mathrm{RF}}\right)$-privacy holds, i.e., receipt-freeness holds in $S$.

One may argue that the choice of $s_{3 N+2}$ and $s_{3 N+3}$ is hardly a strategy for Dan, since the choice is based on the permutation $\psi$ (that neither Dan nor the intruder would know), but formally that is fine since the existential 
quantifier over the $s_{i}$ only requires that there is a recipe that works, and thus our construction is just the simplest way to conduct the proof of receipt-freeness. Dan can choose any vote on the bulletin board that matches the intruder's expectation for Dan $\theta_{I}\left(v_{1}\right)$.

The aspect of strategy becomes even more relevant if we consider the case that more than one voter is bribed by the intruder, because the intruder knows that some agent is lying if more than one points to the same vote. This becomes an issue when the intruder has bribed a significant part of the voters, which may be possible when a vote is held among a small consortium. If the bribed voters have no way to "coordinate" their story, the risk of a collision (that reveals the lie) comes into play.

We observe that our definition of receipt-freeness is independent of what the intruder actually wants: we actually have formalized that agents vote however they want and we prove that they can get away with lying - however only with respect to models of $\alpha$. If the intruder has bribed more voters than actually want to vote for the intruder's preferred choice, then the expected outcome is not a model of $\alpha$ (since the result is not compatible with all bribed people having voted the way the intruder wants). Both this and the previous issue (of coordination) are problems that arise when a significant part of the votership is bribed: they may be coerced into voting what the intruder wants out of fear not to get away with lying after all. These are the boundaries where a possibilistic approach like $(\alpha, \beta)$-privacy makes sense and where probabilities and behavior models would be needed. We see it as a strong point for the declarativity of $(\alpha, \beta)$-privacy that such subtle points become clearly visible from the formalization and discussion of examples.

\subsection{Violation of Receipt-Freeness in FOO'92}

To see the problems of FOO'92 with receipt-freeness, let us consider just the state after the third phase of the protocol. In this case, the bulletin board contains all the ballots (the signed commitments) but the commitment secrets have not yet been revealed. In this state receipt-freeness does not hold: Unopened commitments violate receipt freeness, since the creator of the commitment is in a unique position to prove authorship to the intruder (by revealing the commitment secret). Effectively, this allows the intruder to bribe agents for obtaining, and this is captured by our notion of receipt freeness. This is in particular relevant since voters could refuse to make the last step (the protocol cannot force them, since, by construction, one cannot see who the missing voters are).

While it is intuitively clear that receipt-freeness is violated in this intermediate step, let us prove that it is violated according to our formal definition. Consider the bulletin board without the commitments open, i.e. the same frame as in state $S$ but removing the elements $m_{2 N+1}, \ldots, m_{3 N}$ (the commitment values). Since in this case also the result has not been published yet, we have here $\alpha=v_{1}, \ldots, v_{N} \in\{0,1\}$, i.e. the intruder knows nothing more than there are $N$ binary votes in the game. 
The knowledge of the coerced voter Dan is the same as in the previous subsection, except for removing the entries $d_{2 N+1}, \ldots d_{3 N}$ which contain the $r[\pi[i]]$ that have not yet been published at this point, of course. The intruder again asks Dan to reveal his knowledge as before, which entails that Dan must claim some vote on the bulletin board to be his own and present a fitting commitment secret, namely struct $_{D a n}\left[d_{3 N+3}\right]=r[1]$. Thus the only consistent story that Dan can give for this value is the truth: $s_{3 N+3}=d_{3 N+3}$. That in turn is only consistent with a given intruder hypothesis $\theta_{I} \models \alpha$ if $\theta_{I}\left(v_{1}\right)=\theta_{0}\left(v_{1}\right)$, i.e., it rules out any model in which Dan has voted differently than he actually did. Thus, at this point, Dan has proved to the intruder what he voted.

In fact, this demonstrates how our notion of receipt-freeness is connected to voting secrecy, namely whether the information given by Dan proves anything to the intruder, i.e., whether it allows him to rule out any model $\theta_{I}$ of $\alpha$. Note that this is a very fine notion: receipt-freeness would be violated even in a state where Dan cannot precisely prove what he voted, but only giving the intruder enough information to rule out some model of $\alpha$.

\section{Related work}

This work is based on the framework of $(\alpha, \beta)$-privacy [5], which is in turn based on Herbrand logic 77. As a variant of First-Order Logic, using the ground terms of uninterpreted function symbols as a universe, Herbrand logic is very expressive, e.g. it can axiomatize natural number arithmetic. The main idea of $(\alpha, \beta)$-privacy is to depart from the most popular approach of specifying privacy as bisimilarity of pairs of processes as in $1-4$. Instead, we define privacy as a reachability problem of states, where each state is characterized by (at least) two formulae, namely $\alpha$ giving the public high-level information (like a voting result), and $\beta$ containing all observations that the intruder could make.

While 5 has already defined voting secrecy, this paper gives the first adaption of $(\alpha, \beta)$-privacy to a real-world voting protocol, namely FOO'92 8 . Another core contribution is the formalization of receipt-freeness, namely as a refinement of standard voting secrecy. Here, the high-level information $\alpha$ remains the same (i.e., the same information must be kept private), but the intruder gets extra observations as part of $\beta$ through the interaction with a voter Dan. The most similar work is [6] where voting privacy, receipt-freeness and coercion-resistance have been expressed with observational equivalence (see also 14]). The formalization of these properties rely on labeled bisimilarity of two processes, also proving a hierarchy between these goals. We believe that our formalization in $(\alpha, \beta)$-privacy is more declarative and intuitive, due to its model-theoretic formulation. An interesting question for future work is how the two approaches compare, i.e., whether one can captures anything as an attack that the other does not. If they turned out to be equivalent in some sense instead, then this would indicate that the "right" concept has been hit.

Another question is automation. There are several fragments of bisimilarity for which automation is being developed. However some protocols, even the rela- 
tively simple FOO'92, are hard to analyze fully automatically: for instance, 6 is at the high-level a manual proof, reducing the problem to a static equivalence of two frames (which is then automated). Only in the recent paper [15] a fully automatic analysis of FOO'92 is given. Our focus on a declarative formalization rather than automation concerns allows often for very simple proofs, e.g. in FOO'92 with a hidden preparation, which basically amounts to finding a fitting interpretation for a permutation, which is exactly how one may want to prove such a property manually or in a proof assistant like Isabelle or Coq.

\section{Conclusion}

$(\alpha, \beta)$-privacy was introduced as a simple and declarative way to specify privacy goals and reason about them. We present here the first major use-case using this framework. This use-case illustrates the refined voting privacy goal that we have defined in this work. Indeed, we showed how for any model $\theta$ of $\alpha$, we could step by step construct a model $\mathcal{I}$ of $\beta$. On top of this privacy property, we defined a new property: receipt-freeness. We showed that receipt-freeness implies voting privacy. We illustrated these properties for a voting system, but both privacy and receipt-freeness are actually relevant to a variety of areas, for instance healthcare privacy [16]. Indeed, prescriptions by a medial doctor have similar requirements regarding privacy and even receipt-freeness: for instance, we want to prevent that a doctor could be coerced by a pharmaceutical company to prescribe specific medication, which is actually a receipt-freeness problem.

We are currently investigating coercion resistance as a stronger variant of receipt-freeness, where the intruder can initially determine values for the coerced voter to use. To counter such attacks, one needs protocols with a different setup than FOO'92, allowing re-voting. This also requires to formalize more details about the underlying transition system than we did in this work. In fact, it is part of ongoing work to provide languages, proof strategies and potentially automated tools for specifying and verifying transition systems with $(\alpha, \beta)$ privacy. The idea is here that the formula $\beta$ can be automatically derived from what happens (like message exchanges) and that only $\alpha$ needs to be specified by the modeler, namely indicating at which point which information is deliberately released.

\section{References}

[1] Abadi, M., Blanchet, B., Fournet, C.: The Applied Pi Calculus: Mobile Values, New Names, and Secure Communication. Journal of the ACM, 65(1), pp 1-41. ACM, New-York (2017).

[2] Blanchet, B., Abadi, M., Fournet, C.: Automated Verification of Selected Equivalences for Security Protocols. In: Proceedings of the 20th IEEE Symposium on Logic in Computer Science (LICS 2005), pp 331-340. IEEE (2005). 
[3] Cortier, V., Rusinowitch, M., Zalinescu, E.: Relating two standard notions of secrecy. Logical Methods in Computer Science, 3(3). (2007).

[4] Delaune, S., Ryan, M., Smyth, B.: Automatic Verification of Privacy Properties in the Applied Pi-Calculus. In: Proceedings of IFIPTM 2008: Joint iTrust and PST Conferences on Privacy, Trust Management and Security (IFIPTM'08), IFIP Conference Proceedings, vol. 263, pp. 263-278. Springer, Heidelberg (2008).

[5] Mödersheim, S., Viganò, L.: Alpha-Beta Privacy. ACM Transactions on Privacy and Security. To appear. Preprint available as DTU Compute Technical Report-2018-7 http://imm.dtu.dk/ samo/abpn.pdf. (2018).

[6] Delaune, S., Kremer, S., Ryan, M.: Verifying privacy-type properties of electronic voting protocols. Journal of Computer Security, 17(4), pp 435487. IOS Press (2009).

[7] Hinrichs, T., Genesereth, M.: Herbrand Logic. Technical Report LG-200602, Stanford University, Stanford, CA, USA (2006).

[8] Fujioka, A., Okamoto, T., Ohta, K.: A Practical Secret Voting Scheme for Large Elections. In: Advances in Cryptology - AUSCRYPT '92, LNCS, vol. 718, pp. 244-251. Springer, Heidelberg (1993).

[9] Kremer, S., Ryan, M.: Analysis of an Electronic Voting Protocol in the Applied Pi Calculus. In: Programming Languages and Systems (ESOP 2005). LNCS, vol. 3444, pp 186-200. Springer, Berlin (2005).

[10] Chaum, D.: Untraceable Electronic Mail, Return Addresses, and Digital Pseudonyms. Commun. ACM, 24(2), pp. 84-90. ACM, New-York (1981).

[11] Naor, M.: Bit Commitment Using Pseudo-Randomness. In: Advances in Cryptology - CRYPTO '89, LCNS, vol. 435, pp. 405-419. Springer, Berlin (1990).

[12] Diffie, W., Hellman, M.: New directions in cryptography. IEEE Transactions on Information Theory, 22(6), pp. 644-654. IEEE (1976).

[13] Chadha, R., Cheval, V., Cioâcă, Ş., Kremer, S.: Automated Verification of Equivalence Properties of Cryptographic Protocols. ACM Transactions on Computational Logic, 17(4). ACM, New-York (2016).

[14] Arapinis, M., Liu, J., Ritter, E., Ryan, M.: Stateful applied pi calculus: Observational equivalence and labelled bisimilarity. Journal of Logical and Algebraic Methods in Programming, vol. 89, pp. 95-149. Elsevier Science Publisher, Amsterdam (2017).

[15] Blanchet, B., Smyth, B.: Automated reasoning for equivalences in the applied pi calculus with barriers. Journal of Computer Security, 26(3), pp. :367-422. 2018. 
[16] Dong, N., Jonker, H., Pang, J.: Formal Analysis of Privacy in an eHealth Protocol. In: Computer Security - ESORICS 2012 - 17th European Symposium on Research in Computer Security. LNCS, vol. 7459, pp. 325-342. Springer, Heidelberg (2012).

[17] Okamoto, T.: An electronic voting scheme. In: Proceedings of IFIP World conference on IT Tools, pp 21-30. (1996)

[18] Okamoto, T.: Receipt-free electronic voting schemes for large scale elections. In Proc. 5th Int. Security Protocols Workshop. LNCS, vol. 1361, pp 25-35. Springer (1997).

\section{A Proofs}

\section{Lemma 1 .}

$\mathcal{I} \models \phi_{\text {frame }}($ struct $)$ and $\mathcal{I}=\phi_{\text {frame }}($ concr $)$.

Proof. Following Definition 7 I models the first conjunct of $\phi_{\text {frame }}($ struct) and $\phi_{\text {frame }}($ concr $)$.

It remains to show that $\mathcal{I}$ models the last conjunct of $\phi_{\text {frame }}$ (struct) and $\phi_{\text {frame }}($ concr $)$. Let $f^{n} \in \Sigma_{o p}$. Let $r_{1}, \ldots, r_{n}$ be $n$ recipes in $\left.\mathcal{T}\right\rfloor_{\Sigma_{o p} \cup D}$. Note that $\mathcal{I}\left(r_{i}\right)=\llbracket r_{i} \rrbracket \approx$. It is sufficient to show that $\left.\mathcal{I} \models \operatorname{struct}\left[f\left(r_{1}, \ldots, r_{n}\right)\right]\right)=$ $f\left(\right.$ struct $\left[r_{1}\right], \ldots$, struct $\left.\left[r_{n}\right]\right)$.

$$
\begin{aligned}
& \mathcal{I}\left(\left(\operatorname{struct}\left[f\left(r_{1}, \ldots, r_{n}\right)\right]\right)=\mathcal{I}(\text { struct })\left(\mathcal{I}\left(f\left(r_{1}, \ldots, r_{n}\right)\right)\right)=\mathcal{I}(\text { struct })\left(\llbracket f\left(r_{1}, \ldots, r_{n}\right) \rrbracket \approx\right)\right. \\
& \quad=\llbracket \operatorname{struct}\left\{\left|f\left(r_{1}, \ldots, r_{n}\right)\right|\right\} \rrbracket \approx \text { by Def. } 7 \\
& \quad=\llbracket f\left(\operatorname{struct}\left\{\left|r_{1}\right|\right\}, \ldots, \text { struct }\left\{r_{n} \mid\right\}\right) \rrbracket \approx \\
& \quad=f\left(\llbracket \operatorname{struct}\left\{\left|r_{1}\right|\right\} \rrbracket \approx, \ldots, \llbracket \text { struct }\left\{\left|r_{n}\right|\right\} \rrbracket \approx\right)=f\left(\mathcal{I}(\text { struct })\left(\llbracket r_{1} \rrbracket \approx\right), \ldots, \mathcal{I}(\operatorname{struct})\left(\llbracket r_{n} \rrbracket \approx\right)\right), \\
& \quad \text { by Def. } 7 \\
& \quad=f\left(\mathcal{I}(\operatorname{struct})\left(\mathcal{I}\left(r_{1}\right)\right), \ldots, \mathcal{I}(\operatorname{struct})\left(\mathcal{I}\left(r_{n}\right)\right)\right)=f\left(\mathcal{I}\left(\operatorname{struct}\left[r_{1}\right]\right), \ldots, \mathcal{I}\left(\operatorname{struct}\left[r_{n}\right]\right)\right) \\
& \quad=\mathcal{I}\left(f\left(\operatorname{struct}\left[r_{1}\right], \ldots, \text { struct }\left[r_{n}\right]\right)\right) .
\end{aligned}
$$

Therefore we proved that $\mathcal{I}=\phi_{\text {frame }}($ struct $)$. By a similar reasoning, we prove that $\mathcal{I} \models \phi_{\text {frame }}($ concr $)$. Thus $\mathcal{I} \models \phi_{\text {frame }}($ struct $)$ and $\mathcal{I} \models \phi_{\text {frame }}($ concr $)$.

\section{Lemma 2 ,}

If $\mathcal{I}($ struct $)=\mathcal{I}($ concr $)$ then $\mathcal{I} \models \phi_{\sim}($ struct, concr $)$.

Proof. Suppose $\mathcal{I}($ struct $)=\mathcal{I}$ (concr). Recall that struct and concr have the same domain $D$ so gen $_{\text {struct }}=$ gen $n_{\text {concr }}=$ gen. Let $r$ and $s$ be two recipes in $\mathcal{T}\rfloor_{\Sigma_{o p} \cup D}$. Suppose now that $\mathcal{I} \models \operatorname{struct}[r]=\operatorname{struct}[s]$.

$$
\begin{aligned}
\mathcal{I} \models \text { struct }[r]=\text { struct }[s] & \text { iff } \mathcal{I}(\text { struct })(\mathcal{I}(r))=\mathcal{I}(\text { struct })(\mathcal{I}(s)) \\
& \text { iff } \mathcal{I}(\text { concr })(\mathcal{I}(r))=\mathcal{I}(\text { concr })(\mathcal{I}(s)) \\
& \text { iff } \mathcal{I}=\text { concr }[r]=\text { concr }[s]
\end{aligned}
$$


Thus $\mathcal{I} \models \phi_{\sim}($ struct, concr $)$.

\section{Theorem 1.}

Voting privacy holds in the state $S$.

Proof. First, let us prove that $\mathcal{I}$ (struct) $=\mathcal{I}($ concr $)$. For the struct, we just have to look at the interpretation of $v[\pi[i]]$ and $r[\pi[i]]$ because all the other terms are uninterpreted symbols. For $i \in\{1, \ldots, N\}$,

$$
\begin{aligned}
\mathcal{I}(v[\pi[i]]) & =\mathcal{I}(v)(\mathcal{I}(\pi)(\llbracket i \rrbracket \approx))=\mathcal{I}(v)\left(\llbracket \pi_{I}(i) \rrbracket \approx\right)=\mathcal{I}(v)\left(\llbracket\left(\psi^{-1} \circ \pi_{0}\right)(i) \rrbracket \approx\right) \\
& =\llbracket \theta_{I}\left(v_{\psi^{-1}\left(\pi_{0}(i)\right)}\right) \rrbracket \approx=\llbracket \theta_{0}\left(v_{\pi_{0}(i)}\right) \rrbracket \approx \\
\mathcal{I}(r[\pi[i]]) & =\mathcal{I}(r)(\mathcal{I}(\pi)(\llbracket i \rrbracket \approx))=\mathcal{I}(r)\left(\llbracket \pi_{I}(i) \rrbracket \approx\right)=\mathcal{I}(r)\left(\llbracket\left(\psi^{-1} \circ \pi_{0}\right)(i) \rrbracket \approx\right) \\
& =\llbracket r_{\left(\psi \circ \psi^{-1} \circ \pi_{0}\right)(i)} \rrbracket \approx=\llbracket r_{\pi_{0}(i)} \rrbracket \approx .
\end{aligned}
$$

Since for the concr, the messages are of the form $m_{N+i} \mapsto \operatorname{verify}(\operatorname{priv}(A)$, commit ( $\left.\left.\theta_{0}\left(v_{\pi_{0}(i)}\right), r_{\pi_{0}(i)}\right)\right)$, we have $\mathcal{I}($ struct $)=\mathcal{I}($ concr $)$. Then we have shown that for every model $\theta_{I} \models \alpha$, i.e. any possible intruder's hypothesis, we can find a model $\mathcal{I}$ of $\beta$ that agrees with $\theta_{I}$, i.e. $\mathcal{I}(v[i])=\theta_{I}\left(v_{i}\right)$ for all votes $v_{i}$.

\section{Theorem 2,}

Voting privacy holds in the state $S^{\prime}$.

Proof. (Sketch) As a first step, let us extend the two frames by the messages that the intruder can deduce by decomposition steps:

$$
\begin{aligned}
& \text { struct }=\left\{\ldots, m_{N+1}^{\prime} \mapsto \operatorname{commit}(v[\pi[1]], r[\pi[1]]), \ldots,\right. \\
& m_{2 N}^{\prime} \mapsto \operatorname{commit}(v[\pi[N]], r[\pi[N]]), m_{2 N+1}^{\prime} \mapsto v[\pi[1]], \ldots, m_{3 N}^{\prime} \mapsto v[\pi[N]], \\
& \left.m_{3 N+1}^{\prime} \mapsto \operatorname{blind}\left(\operatorname{commit}(v[1], r[1]), b_{1}\right), \ldots, m_{4 N}^{\prime} \mapsto \operatorname{blind}\left(\operatorname{commit}(v[N], r[N]), b_{N}\right)\right\}
\end{aligned}
$$

No further subterms can be obtained by decomposition, and the checks that verifiers give us are analogous between $\mathcal{I}$ (struct) and $\mathcal{I}$ (concr).

Consider any two equivalent terms $s \approx t$ that do not contain destructors or verifiers. While $\approx$ allows to reorder signatures and blindings, it cannot remove or introduce any signature or blinding, i.e., if verify $(k, m)$ is a subterm of $s$, then there is a subterm verify $\left(k, m^{\prime}\right)$ of $t$ (modulo $\approx$ ) for some term $m^{\prime}$. Similar statements hold for blind and commit.

Suppose now two recipes $r_{1}$ and $r_{2}$ that only contain constructors (verify, blind, commit) and labels, and suppose $\mathcal{I}\left(\right.$ struct $\left.\left[r_{1}\right]\right)=\mathcal{I}\left(\right.$ struct $\left.\left[r_{2}\right]\right)$. We show that then also $\mathcal{I}\left(\operatorname{concr}\left[r_{1}\right]\right)=\mathcal{I}\left(\operatorname{concr}\left[r_{2}\right]\right)$. (The proof for the other direction is similar.)

The proof is by induction over the maximum depth of $r_{1}$ and $r_{2}$ where $\operatorname{depth}\left(m_{i}\right)=0$ and $\operatorname{depth}\left(f\left(t_{1}, \ldots, t_{n}\right)\right)=1+\max _{i=1}^{n}\left(\operatorname{depth}\left(t_{i}\right)\right)$. For depth 0 , i.e., two labels, it is trivial: for two distinct labels the result is the same only on plain votes (i.e. $\left.m_{2 N+i}^{\prime}\right)$, and here $\mathcal{I}($ concr $)=\mathcal{I}($ struct $)$. 
Suppose now the statement holds for all $r_{1}^{\prime}, r_{2}^{\prime}$ up to depth $\mathfrak{N}$, we show that it also holds for all $r_{1}$ and $r_{2}$ up to depth $\mathfrak{N}+1$. We proceed by case distinction (omitting symmetric cases).

1. $r_{1}$ is a label and $r_{2}$ is of depth $\mathfrak{N}+1$ (otherwise it is already covered). Thus $r_{2}$ starts with a constructor, which we can distinguish:

- $r_{2}=\operatorname{verify}\left(r^{\prime}, r^{\prime \prime}\right)$. Now $\mathcal{I}\left(\right.$ struct $\left.\left[r^{\prime}\right]\right)$ is a term that the intruder uses as a signing key and that is a known term to him. Note that no message contains a signature with a signing key known to the intruder. It is thus actually impossible that $\mathcal{I}\left(\operatorname{struct}\left(r_{1}\right)\right)=\mathcal{I}\left(\operatorname{struct}\left(r_{2}\right)\right)$, since signatures cannot disappear in constructor terms.

- A similar proof shows that $r_{2}=\operatorname{commit}\left(r^{\prime}, r^{\prime \prime}\right)$ and $r_{2}=\operatorname{blind}\left(r^{\prime}, r^{\prime \prime}\right)$ is impossible.

2. Both $r_{1}$ and $r_{2}$ are composed, at least one, say $r_{1}$, is of depth $\mathfrak{N}+1$. Again let us consider the case that $r_{1}=\operatorname{verify}\left(r_{1}^{\prime}, r_{2}^{\prime \prime}\right)$, the proof for commit and blind is similar.

Again, since signatures cannot disappear in constructor terms, $\mathcal{I}\left(\right.$ struct $\left.\left[r_{2}\right]\right)$ must contain a subterm verify $(k, m)$ with $k=\mathcal{I}\left(\right.$ struct $\left.\left[r_{1}^{\prime}\right]\right)$. Since $k$ is a term the intruder can construct, and he knows no key that has been used for signing in his knowledge, this signature was constructed by the intruder in $r_{2}$ as well. Thus $r_{2} \approx \operatorname{verify}\left(r_{2}^{\prime}, r_{2}^{\prime \prime}\right)$ such that $\mathcal{I} \models \operatorname{struct}\left[r_{1}^{\prime}\right]=$ $\operatorname{struct}\left[r_{2}^{\prime}\right] \wedge \operatorname{struct}\left[r_{1}^{\prime \prime}\right]=\operatorname{struct}\left[r_{2}^{\prime \prime}\right]$. Since $r_{1}^{\prime}, r_{2}^{\prime}, r_{1}^{\prime \prime}$, and $r_{2}^{\prime \prime}$ are all of size smaller or equal to $\mathfrak{N}$, so we can apply the induction hypothesis and conclude $\mathcal{I} \models \operatorname{concr}\left[r_{1}^{\prime}\right]=\operatorname{concr}\left[r_{2}^{\prime}\right] \wedge \operatorname{concr}\left[r_{1}^{\prime \prime}\right]=\operatorname{concr}\left[r_{2}^{\prime \prime}\right]$ and thus $\mathcal{I}=\operatorname{concr}\left[r_{1}\right]=\operatorname{concr}\left[r_{2}\right]$.

\section{Theorem 3 .}

$\left(\alpha, \beta_{\mathrm{RF}}\right)$-privacy holds, i.e., receipt-freeness holds in $S$.

Proof. The idea once again here is to prove that for all $\theta_{I}=\alpha, \mathcal{I}($ struct $)=$ $\mathcal{I}($ concr $)$. We extend the proof of Theorem 1. gen is extended to the domain $D \cup D_{D a n}$. The frames struct and concr are also extended to the new domain as explained with the knowledge of Dan. We already described Dan's strategy for lying. By definition, $\mathcal{I}(v[1])=\llbracket \theta_{I}\left(v_{1}\right) \rrbracket \approx$ and $\mathcal{I}(r[1])=\llbracket r_{\psi(1)} \rrbracket \approx$

Since $\mathcal{I}\left(\operatorname{concr}_{3}\left[d_{3 N+2}\right]\right)=\mathcal{I}\left(\right.$ concr $\left._{D a n}\left[s_{3 N+2}\right]\right)=\llbracket \theta_{0}\left(v_{\psi(1)}\right) \rrbracket \approx=\llbracket \theta_{I}\left(v_{1}\right) \rrbracket \approx$ and $\mathcal{I}\left(\right.$ concr $\left.\left[d_{3 N+3}\right]\right)=\mathcal{I}\left(\right.$ concr $\left._{\operatorname{Dan}}\left[s_{3 N+3}\right]\right)=\llbracket r_{\psi(1)} \rrbracket \approx$ by construction, we still have $\mathcal{I}($ struct $)=\mathcal{I}($ concr $)$. Thus, in the augmented state $S$, receipt-freeness holds. 